\title{
MELHORIA DA EFICIÊNCIA ENERGÉTICA DE REFRIGERADORES DOMÉSTICOS
}

\author{
Edson A. Vendrusculo e José A. Pomilio \\ Faculdade de Engenharia Elétrica e de Computação - UNICAMP \\ Departamento de Sistemas e Controle de Energia - Laboratório de Condicionamento de Energia Elétrica \\ CEP 13083-970 - C. P. 6101 - Campinas - SP - Brasil \\ e-mail: \{ adriano, antenor $\} @$ dsce.fee.unicamp.br
}

\begin{abstract}
Resumo - Este trabalho propõe a ação conjunta de um acionamento eletrônico e um controle de temperatura visando aumentar a eficiência energética e melhorar a qualidade da energia elétrica de um refrigerador popular. Um refrigerador com capacidade de armazenamento de aproximadamente 300 l é considerado, devido sua predominância em famílias de baixa renda. A redução da variação de temperatura nos trocadores de calor foi validada como estratégia para o aumento da eficiência energética de refrigeradores domésticos. Simulações indicam a melhoria na resposta dinâmica da temperatura em função do ajuste dos ganhos de escalonamento de um compensador Fuzzy-PI. Um modelo de primeira ordem foi obtido a partir do ajuste de funções exponenciais temporais a um perfil, obtido experimentalmente, da resposta dinâmica de temperatura. A otimização do conjugado mecânico e o alto fator de potência foram experimentalmente comprovados através de um conversor estático de potência, o qual dispõe de um link CC comum aos estágios ativos de entrada e saída, e aciona um motor/compressor monofásico assimétrico.
\end{abstract}

Palavras-chave - Compensador Fuzzy, Consumo de Energia, Eficiência Energética, Etiquetagem, Refrigerador, Trocadores de Calor.

\section{ENERGY EFFICIENCY IMPROVEMENT OF RESIDENTIAL REFRIGERATORS}

Abstract - This paper purposes a electronic driving linked to a temperature control strategy aiming to increase the energy efficiency and to improve the power quality issues of a popular refrigerator. In such a case, the net capacity of the refrigerator is lower than 320liters. It was verified that the energy efficiency might be increased using a Fuzzy PI controller. Scaling gains added to input and output variables of the Fuzzy-PI controller improve the temperature dynamic response. The plant model is of first order and encompasses a set of exponential functions that represents the temperature evolution inside the evaporator. A driving system composed of a front-end converter, dc link and inverter changes the motor-compressor frequency. The front-end converter provides power factor correction through a

\footnotetext{
Artigo submetido em 15 de maio de 2008; primeira revisão em 03 de outubro de 2008; segunda revisão em 12 de novembro de 2008. Recomendado para publicação pelos Editores da Seção Especial, João Carlos dos Santos Fagundes e Felix Alberto Farret.
}

controlled voltage doubler with a series inductor. The inverter has two legs and the middle point of the dc link connects the common point of the split-phase motor/compressor. It was verified experimentally that the electricity consumption decreases because of the reduction in the temperature ripple at heat exchangers.

Keywords - Electricity Consumption, Energy Efficiency, Fuzzy-PI Compensator, Heat Exchangers, Labeling, Refrigerator.

\section{INTRODUÇÃO}

Em 17 de outubro de 2001 foi instituída a Lei 10.295, conhecida como Lei de Eficiência Energética, a qual delega ao Poder Executivo o estabelecimento dos índices mínimos de eficiência energética de máquinas e aparelhos consumidores de energia fabricados e comercializados no País [1]. Estabelece ainda que, num prazo de até 1 ano a partir da regulamentação específica de cada produto, um programa de metas deve ser elaborado, para uma progressiva evolução dos índices. Em consequiência, e sob coordenação do INMETRO, foi consolidado o Programa Brasileiro de Etiquetagem (PBE), o qual é um mecanismo de avaliação da conformidade compulsória na área de desempenho energético. Os produtos aprovados no PBE estão autorizados a ostentar a Etiqueta Nacional de Conservação de Energia (ENCE). A etiqueta, específica para cada linha de eletrodoméstico, indica a eficiência energética do equipamento em uma escala decrescente, representada por faixas coloridas indicadas pelas letras de $\mathrm{A}$ (mais eficiente) até G, conforme ilustra a Figura 1.

$\mathrm{O}$ refrigerador demanda o maior percentual de consumo de energia elétrica nos domicílios brasileiros [1] e, em relação aos índices europeus, detém uma eficiência até $50 \%$ menor. Por exemplo, refrigeradores de ambas as nacionalidades e com características semelhantes, ou seja, mesma capacidade e pertencente à categoria uma (1) estrela, segundo a norma ISO7371 [2], tem consumo de 24,8 kWh/mês [3] e 13,1 $\mathrm{kWh} /$ mês [4], respectivamente. Ações de política pública e de fomento à inovação tecnológica são mecanismos utilizados para melhorar o desempenho energético. Neste contexto, este artigo apresenta resultados experimentais da eficiência energética de um refrigerador de uma porta, popular ${ }^{1}$, pertencente à classe 1 estrela, utilizando um controlador de temperatura com lógica Fuzzy.

\footnotetext{
${ }^{1} \mathrm{O}$ termo "popular" corresponde ao tipo de refrigerador com o maior percentual de comercialização no mercado nacional [1], predominantemente adquirido pela população de baixa renda [7].
} 


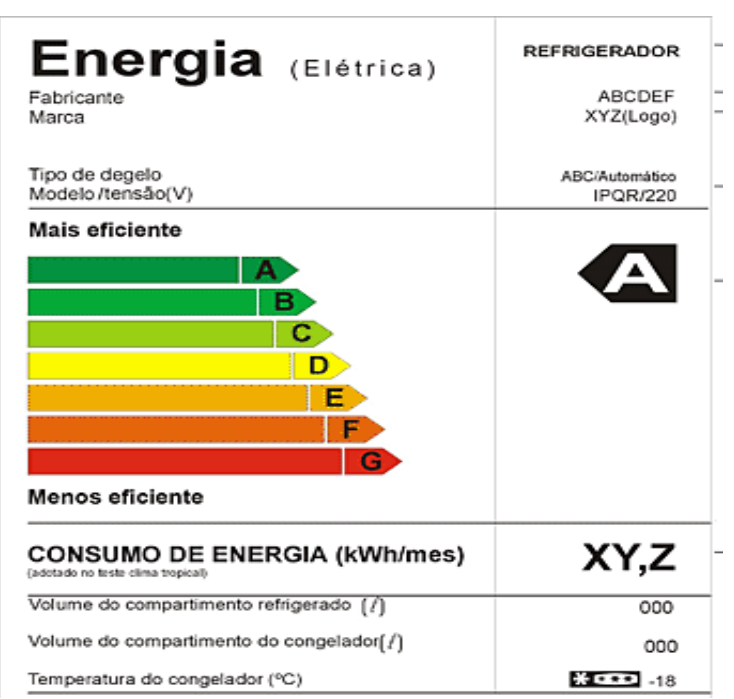

Fig. 1. Etiqueta de eficiência energética para refrigeradores.

Trabalhos científicos, sobre aplicação de lógica Fuzzy em eletrodomésticos, abordam somente aspectos gerais do controle, uma vez que cada tipo de eletrodoméstico tem comportamento peculiar [5]. A aplicação da teoria Fuzzy, na síntese somente do controlador de temperatura, tem se mostrado uma solução parcial resultando em um aumento não significativo de eficiência energética [6]. Logo, neste trabalho o controlador Fuzzy contempla os limites da planta no acionamento do motor/compressor, permitindo a otimização das condições de operação do sistema de refrigeração.

De modo geral, refrigeradores com eletrônica embutida, utilizam um estágio de entrada constituído por retificadores a diodos com filtro capacitivo, e uma técnica de controle de temperatura não disponível ao público, devido à elevada competitividade do mercado. Portanto, neste trabalho o conversor estático de potência dispõe de um estágio de entrada ativo, o qual garante alto fator de potência e regulação da tensão no link CC. Este último é comum ao estágio de saída, o qual consiste de um inversor com dois braços ativos, conectados aos enrolamentos auxiliar e principal do motor/compressor. Cabe notar que comercialmente conversores estáticos de potência são empregados em refrigeradores não acessíveis pela população de baixa renda, a qual representa $37 \%$ da população brasileira, e adquire refrigeradores com capacidade igual ou menor que 320 litros [7].

Atualmente, os refrigeradores nacionais de baixo custo utilizam um controle on-off de temperatura, imposto por um termostato mecânico. Esta estratégia mantém a temperatura média dentro dos limites desejados. Contudo, uma elevada variação de temperatura é experimentada pelos elementos trocadores de calor, ou seja, evaporador e condensador, diminuindo o coeficiente de desempenho do sistema de refrigeração. A estratégia de controle proposta neste artigo visa reduzir a variação da temperatura nos trocadores de calor ${ }^{2}$, atuando na frequiência de excitação do compressor.

\footnotetext{
${ }^{2}$ É possível demonstrar analiticamente que a operação do sistema de refrigeração em temperaturas de evaporação maiores melhora o coeficiente de desempenho.
}

\section{CLASSIFICAÇÃO DO REFRIGERADOR E CARAC- TERÍSTICAS DO COMPRESSOR HERMÉTICO}

O refrigerador de uma porta utilizado pertence à classe 1 estrela da norma ISO7371,ou seja, a temperatura nominal de classificação é $-6{ }^{\circ} \mathrm{C}$. Portanto, a maior temperatura medida no compartimento denominado congelador $\mathrm{T}_{c}$ deve atender os limites $-12{ }^{\circ} \mathrm{C}<\mathrm{T}_{\mathrm{c}} \leq-6{ }^{\circ} \mathrm{C}$. A temperatura média no compartimento para os alimentos refrigerados (fresh-food) deve ser mantida em $5{ }^{\circ} \mathrm{C}$. A norma ISO 7371 especifica os procedimentos para a medição do consumo de energia mantendo a porta fechada e a temperatura e a umidade ambiente, respectivamente, em $32^{\circ} \mathrm{C}$ e $50 \%$, dentro da câmara térmica.

Normalmente, os compressores utilizados em HVAC (heating, ventilation and air-conditioning) contêm motores com dois enrolamentos assimétricos, alimentados a partir de uma fonte monofásica conforme ilustra a Figura 2.

Três terminais identificam os enrolamentos: $\mathbf{M}$ (bobina de marcha ou enrolamento principal -"main"), $\mathbf{S}$ (bobina de partida ou enrolamento auxiliar - "start") e o ponto comum C a ambos os enrolamentos. Um campo girante é obtido devido ao deslocamento de 90 graus elétricos dos enrolamentos, obtido construtivamente na distribuição física das ranhuras em volta do estator, e ao defasamento no tempo (não necessariamente de 90 graus) entre as correntes elétricas, obtido através do dimensionamento de enrolamentos com impedâncias distintas. Devido à possibilidade de variações na sua impedância, e no tipo de conexão do enrolamento auxiliar à rede elétrica, valores distintos de conjugados podem ser obtidos, originando diversas denominações quanto ao uso de compressores monofásicos.

A Figura 2 ilustra o compressor com princípio de funcionamento RSIR (Split-Phase ou Resistance Start/Induction Run), o qual pode ter um capacitor adicionado em série com o enrolamento auxiliar originando o princípio CSIR (Capaci-

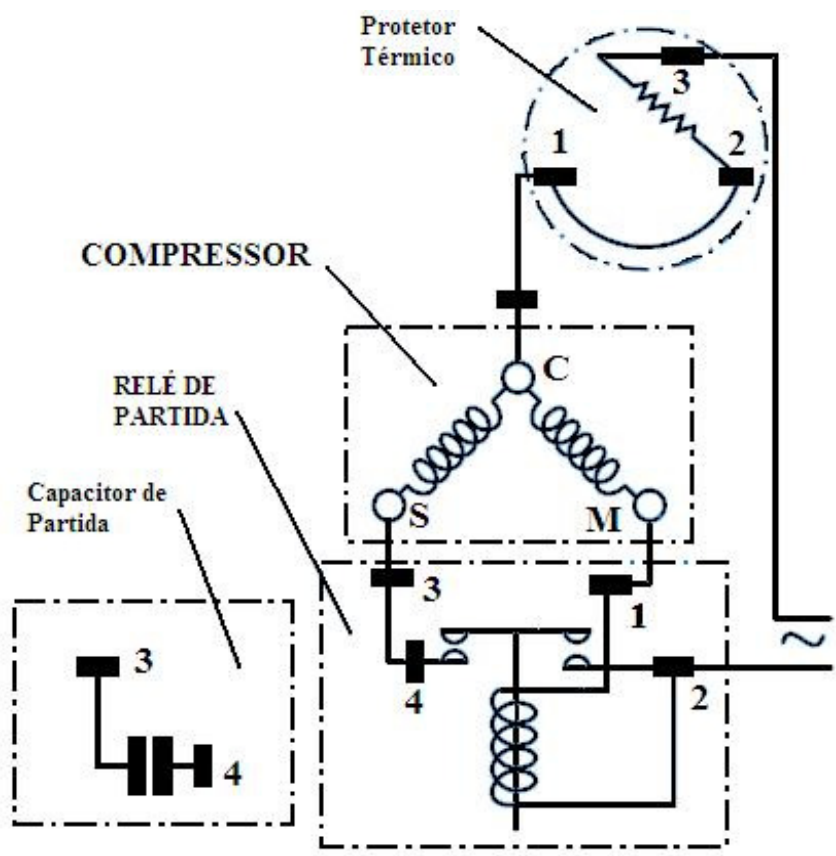

Fig. 2. Diagrama elétrico de conexão de um compressor hermético RSIR/CSIR. 
tor Start/Induction Run). Quando o relé de partida (Figura 2), for substituído por um relé do tipo PTCR (Positive Coefficient Temperature Resistor), obtém-se o princípio PTCSIR (Start by PTC / Induction Run) ou PTCCSIR (Start by PTC / Capacitor Start Induction Run). Outros princípios são obtidos se um capacitor permanente é empregado. Neste trabalho foi utilizado um compressor com princípio $\mathrm{RSIR}^{3}$ o qual tem o menor custo de mercado [8]. O mesmo tem um conjugado normal de partida e é adequado para aplicações em sistemas de refrigeração com baixa pressão de retorno.

\section{CONVERSOR ESTÁTICO COM ESTÁGIOS ATIVOS DE ENTRADA E SAÍDA}

O conversor utilizado para o acionamento do compressor dispõe de um número reduzido de chaves e permite obter alto fator de potência com conseqüente aumento da tensão no link CC, conforme sugere a Figura 3 [9].

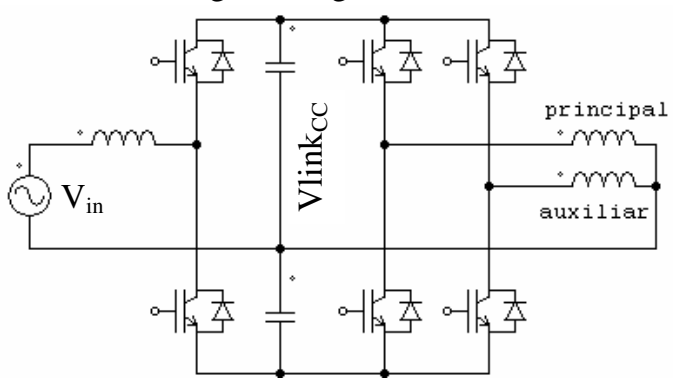

Fig. 3. Conversor para acionamento de motor monofásico com enrolamentos assimétricos.

Os parâmetros que delimitam o projeto do conversor são a relação de espiras e a defasagem entre as correntes nos enrolamentos da máquina. Para manter um fluxo rotacional com trajetória perfeitamente circular é necessário manter a corrente no enrolamento auxiliar 90 graus adiantada em relação àquela do enrolamento principal e garantir a mesma força magnetomotriz em ambos os enrolamentos, ou seja, manter a relação:

$$
n=I_{\text {main }} / \mathrm{I}_{\text {aux }}
$$

sendo $n$ a relação de espiras entre os enrolamentos auxiliar e principal e $I_{\text {aux }}$ e $I_{\text {main }}$ as respectivas correntes. Isto implica manter a seguinte relação entre as tensões produzidas pelo inversor, fonte de tensão:

$$
n=V_{\text {aux }} / \mathrm{V}_{\text {main }}
$$

Uma vez que $n>1$ o link CC deve ter uma tensão adequada para atingir o valor de pico necessário para o enrolamento auxiliar. Logo, o conversor apresentado na Figura 3 deve produzir uma tensão mínima no link CC calculada por:

$$
V_{\text {linkCC }}=2 \sqrt{2} \cdot n \cdot V_{\text {in }}
$$

\footnotetext{
3 Características elétricas: Referência comercial: 1/10 HP; Tensão/Freqüência: 115 V/60 Hz; Corrente: 1,3 A; Potência:98 W.

Características mecânicas: Compressor tipo: recíproco; Deslocamento: 3,77 $\mathrm{cm}^{3}$; Baixo conjugado de partida: LST; Tipo de aplicação: Baixa Pressão de Retorno (LBP); Refrigerante: R134a; Elemento de Controle: Tubo Capilar; Pressão Alta: 1600kPa; Pressão Baixa: 590kPa.
}

sendo $\mathrm{V}_{\text {in }}$ o valor eficaz da tensão da rede [9].

Na implementação do controle do conversor a equalização da tensão no link CC é garantida medindo a tensão em cada capacitor, e a diferença entre ambas é adicionada ao sinal senoidal de referência de corrente.

Adicionalmente, devido ao valor finito das capacitâncias durante a carga dos capacitores, a qual ocorre a cada semiciclo da rede, existem componentes harmônicos de ordem par na tensão total no link CC. Se a tensão realimentada do link CC não for filtrada haverá uma distorção (através da adição de um terceiro harmônico) e também um deslocamento de fase na corrente absorvida da rede, reduzindo o fator de potência. Portanto, o sinal de tensão da rede é adicionado ao sinal modulante (feed forward) do PWM (modulação por lagura de pulso) para garantir o alto fator de potência [10].

\section{CARACTERIZAÇÃO DO PERFIL DE TEMPERATURA}

As propriedades termodinâmicas e a interação entre os elementos de um sistema de refrigeração impedem que a resposta dinâmica, devido a alterações na entrada, seja independente de condições de operação iniciais específicas. Ou seja, perturbações idênticas, provocadas em diferentes condições iniciais (em regime permanente), não causarão respostas de mesma amplitude [11]. Logo, ensaios em laboratório foram executados para verificar o comportamento da temperatura em função do acionamento on-off do compressor. A Figura 4 ilustra os elementos principais do sistema de refrigeração, ou seja, condensador, evaporador, compressor e tubo capilar (ou válvula de expansão).

O compressor além de bombear o fluido refrigerante, aumenta a diferença de pressão e temperatura entre o condensador (alta pressão) e o evaporador (baixa pressão). Em contrapartida, o tubo capilar atua como limitador de vazão do sistema. A temperatura no evaporador (sensor fixado em contato com o material alumínio) varia de $-25{ }^{\circ} \mathrm{C}$ a $-7{ }^{\circ} \mathrm{C}$, sendo o valor médio mantido em $-15^{\circ} \mathrm{C}$ pelo controle on-off. Portanto, uma variação de $18{ }^{\circ} \mathrm{C}$ é imposta a este elemento, enquanto o condensador experimenta uma variação de $23{ }^{\circ} \mathrm{C}$.

A temperatura do ar frio dentro do evaporador é a variável

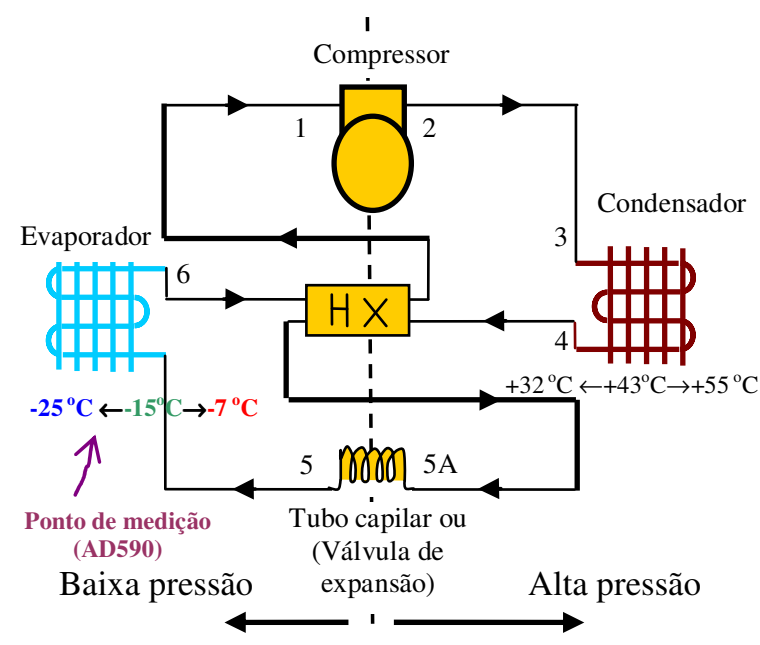

Fig. 4. Elementos de um sistema de refrigeração e temperatura nos trocadores de calor. 
controlada e foi medida usando um sensor de temperatura AD590. A conversão dos valores de tensão indicados na Figura 5 (escala vertical) para valores absolutos de temperatura é feita considerando a função de transferência estática do sensor, ou seja:

$$
T\left[{ }^{\circ} C\right]=\frac{\text { Volts }}{0,01}-273,15
$$

Sendo Volts a tensão medida e $T$ a temperatura. $\mathrm{O}$ sensor opera como fonte de corrente controlada por temperatura. A precisão é de $1 \mu \mathrm{A} / \mathrm{K}(\mathrm{K}=273,15+\mathrm{C})$, $\mathrm{K}$ representando graus na escala Kelvin e $\mathrm{C}$ graus na escala Celsius. A temperatura no ar dentro do evaporador estabiliza no valor médio de -6 ${ }^{\circ} \mathrm{C}$ (atendendo a norma ISO7371) e atinge valores máximos e mínimos de $+3{ }^{\circ} \mathrm{C} \mathrm{e}-15^{\circ} \mathrm{C}$, respectivamente. Isto caracteriza uma variação de $18^{\circ} \mathrm{C}$.

A Figura 5 apresenta o perfil da temperatura dentro do evaporador de onde é possível inferir que o controle on-off mantém o compressor ligado durante 8 minutos e desligado por 12 minutos, portanto resultando num ciclo de trabalho de $40 \%$. Estes valores dependem da condição ambiente onde está situado o refrigerador, tal como temperatura e umidade.

$O$ perfil da variação da temperatura, em função do acionamento do compressor, identificado na Figura 5 pelo decréscimo de temperatura, foi utilizado para obter um modelo que permita a simulação do sistema. Considerando uma soma de funções temporais exponenciais, a função $\mathrm{y}(t)$ que representa o comportamento dinâmico pode ser definida por:

$$
y(t)=y(\infty)+A e^{-\alpha . t}+B e^{-\beta . t}+C e^{-\gamma . t}+\ldots .
$$

Devido ao perfil monotônico somente uma função exponencial foi considerada, ou seja:

$\hat{y}_{\text {deg rau }}(t)=\left(258,15+20,15 \cdot e^{-0,004 . t}\right)-273,15\left[{ }^{\circ} \mathrm{C}\right]$

Portanto, aplicando a Transformada de Laplace e rearranjando os termos resulta:

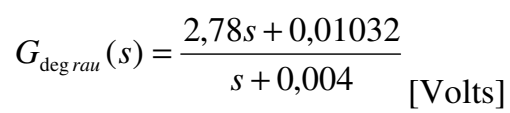

A função expressa por (7) representa a planta e é utilizada na simulação do sistema de refrigeração com controle Fuzzy discutido na próxima seção.

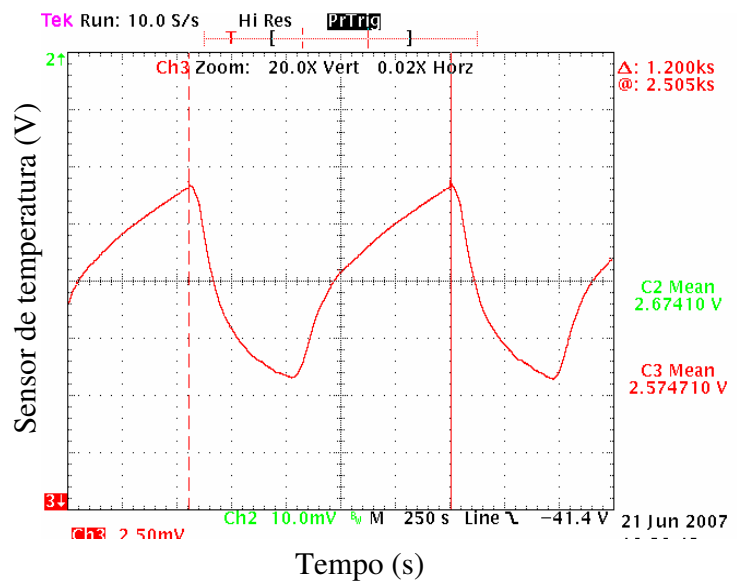

Fig. 5. Dinâmica da temperatura no evaporador.
Considerações sobre o comportamento e limitações da planta (sistema de refrigeração)

Controladores Fuzzy são desenvolvidos baseados no conhecimento do operador a respeito das reações do processo face as suas ações. No caso do refrigerador, o comportamento dinâmico das temperaturas e os limites mecânicos do compressor/refrigerador precisam ser conhecidos. Alguns detalhes peculiares e considerações sobre o sistema são apontados a seguir:

- Devido à presença do tubo capilar a diferença de pressão entre a saída do condensador e a entrada do evaporador é gradualmente atenuada ao longo do próprio tubo. Desta forma, o fluido refrigerante circula livremente assim que o compressor é acionado. Isto caracteriza uma resposta praticamente imediata (sem tempo morto) na variação da temperatura.

- Compressores que operam com velocidade (ou rotação) variável são tecnicamente definidos como compressores de capacidade variável (Variable Capacity Compressor), e podem ter sua eficiência volumétrica alterada em função da rotação. Neste estudo é considerado que a eficiência volumétrica permanece constante para todo o universo de discurso estabelecido para a variável de saída do controlador fuzzy, a qual afeta diretamente a rotação do compressor.

- Os limites máximo e mínimo do universo de discurso da variável de saída são definidos em função de limitações mecânicas. Ou seja, ineficácia na lubrificação interna do compressor para rotações menores que 2000RPM e problemas mecânicos estruturais para rotações elevadas (acima de $4500 \mathrm{rpm})$.

- Uma vez que existe um limite mínimo de rotação, devido a problemas de lubrificação, o compressor deve ser desligado caso a porta do refrigerador não seja aberta por um longo tempo e/ou nenhuma carga térmica seja colocada dentro do mesmo. Em outros termos, se o controlador fuzzy diminuir continuamente a rotação, devido ao excesso de frio, a mínima rotação é atingida e a partir deste momento é necessário desligar o compressor para evitar sobreresfriamento.

- O refrigerador contém um compressor/motor do tipo SplitPhase ou Resistance Start/Induction Run (RSIR). O conjugado de partida é adequado para aplicações em sistemas de refrigeração que operam com baixa pressão de retorno, ou seja, que utilizam tubo capilar como dispositivo de controle de fluxo. Esta característica impõe restrições na partida do compressor, ou seja, depois de desligar o compressor é necessário aguardar um tempo mínimo para que ocorra a total "equalização" de pressão ao longo do tubo capilar. Caso contrário, o motor/compressor pode ser sobrecarregado devido à insuficiência de conjugado de partida. 


\section{CONTROLADOR PI-FUZZY}

A lógica Fuzzy foi utilizada para avaliar um controlador com ações PI considerando os ganhos de escalonamento $\mathrm{g}_{\mathrm{a}}$, $\mathrm{g}_{\mathrm{b}}$ e $\mathrm{g}_{\mathrm{c}}$ de entrada e saída. O controlador Fuzzy-PI foi derivado do controlador clássico tipo PI definido por:

$$
u_{p i}=K_{P} \cdot e+K_{I} \cdot \int e \cdot d t
$$

Sendo $u_{p i}$ a saída, $e$ a entrada (erro), $K_{P}$ o ganho proporcional, e $K_{I}$ o ganho integral. Colocando em evidência o ganho proporcional em (8) resulta:

$$
u_{p i}=\int\left\{K_{P}\left(\dot{e}+T_{I} \cdot e\right)\right\} \cdot d t
$$

A constante de tempo do controlador é definida como $T_{I}=K_{P} / K_{I}$. Considerando $\boldsymbol{e}$ e $\dot{e}$ (taxa de variação do erro) as variáveis de entrada para um conjunto de regras Fuzzy o controlador no domínio contínuo é representado pelo diagrama de blocos ilustrado da Figura 6.a.

A representação no domínio discreto pode ser obtida a partir da análise do diagrama de blocos, ou seja, a variação do erro $(d e / d t=\dot{e})$ é descrita por:

$$
\dot{e} \rightarrow \Delta e(k)=e(k)-e(k-1)=\left(1-z^{-1}\right) e(k)
$$

Uma vez que o bloco Fuzzy recebe como entrada a variação $\Delta e(k)$, a função de transferência discreta com entrada $e(k)$ é:

$$
\frac{\Delta e(k)}{e(k)}=1-z^{-1}
$$

Para o controlador com ação integral a saída do bloco Fuzzy é a variação $\Delta u$ e a saída do controlador propriamente é a variável $u$. Então no domínio discreto resulta:

$$
\Delta u(k)=u(k)-u(k-1)=\left(1-z^{-1}\right) u(k)
$$

Logo, a função de transferência na saída é dada por:

$$
\frac{u(k)}{\Delta u(k)}=\frac{1}{1-z^{-1}}
$$

Resultando o diagrama de blocos para o controlador discreto PI-Fuzzy indicado na Figura 6.b. A relação entre os ganhos de escalonamento do controlador PI-Fuzzy e os ganhos proporcional e integral dos controladores contínuos é dado por [12]:

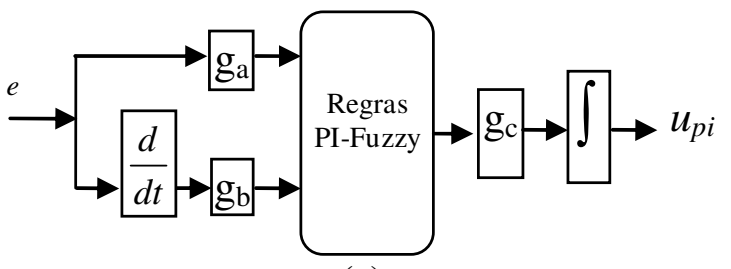

(a)

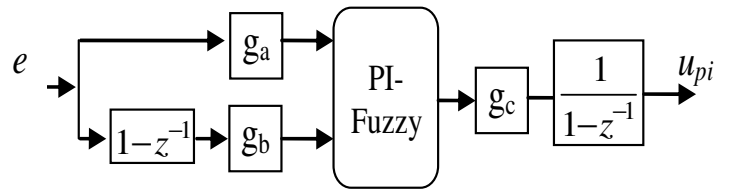

(b)

Fig. 6. Diagrama de blocos do controlador PI-Fuzzy contínuo (a) e discreto (b).

$$
\begin{aligned}
& K_{P}=g_{c} F\left(\frac{g_{b}}{T}\right) T \\
& K_{I}=g_{c} F\left(g_{a}\right) T
\end{aligned}
$$

Sendo que $F($.$) representa uma operação Fuzzy, ou seja, o$ mapeamento não linear estático entre as entradas e saídas do bloco Fuzzy. Os efeitos dos ganhos $K_{P}$ e $K_{I}$ no comportamento de um sistema são facilmente compreendidos pelos projetistas de controle, logo as relações anteriores (14) e (15) são úteis para o ajuste do ganho dos controladores Fuzzy.

\section{CONSIDERAÇÕES SOBRE A SIMULAÇÃO E IM- PLEMENTAÇÃO DO COMPENSADOR PI-FUZZY APLICADO AO CONTROLE DE TEMPERATURA}

Para testar a técnica Fuzzy uma simulação em Simulink/Matlab do sistema (refrigerador) foi realizada reproduzindo os ganhos e condições encontradas no sistema real que utiliza um DSP TMS320F2812, o sensor de temperatura AD590 e um refrigerador de 320 litros de capacidade, conforme ilustra a Figura 7. No diagrama, os blocos que estão no domínio discreto, emulando a implementação no processador, são aqueles compreendidos entre o bloco ZOH (zeroorder hold) e o bloco denominado integral, inclusive.

O modelo da planta é aquele obtido anteriormente (7), ou seja, a resposta para um degrau de $0 \mathrm{a} 60 \mathrm{~Hz}$ na freqüência de excitação do motor/compressor. $\mathrm{O}$ atraso de 2 segundos foi considerado, contudo para sistemas com tubo capilar este atraso é desprezível quando comparado a constante de tempo térmica do refrigerador. Os ganhos do sensor AD590 igual a 100 e na digitalização do conversor analógico-digital $(3 \mathrm{~V}=$ 1 p.u na variável interna do DSP) são representados pelo

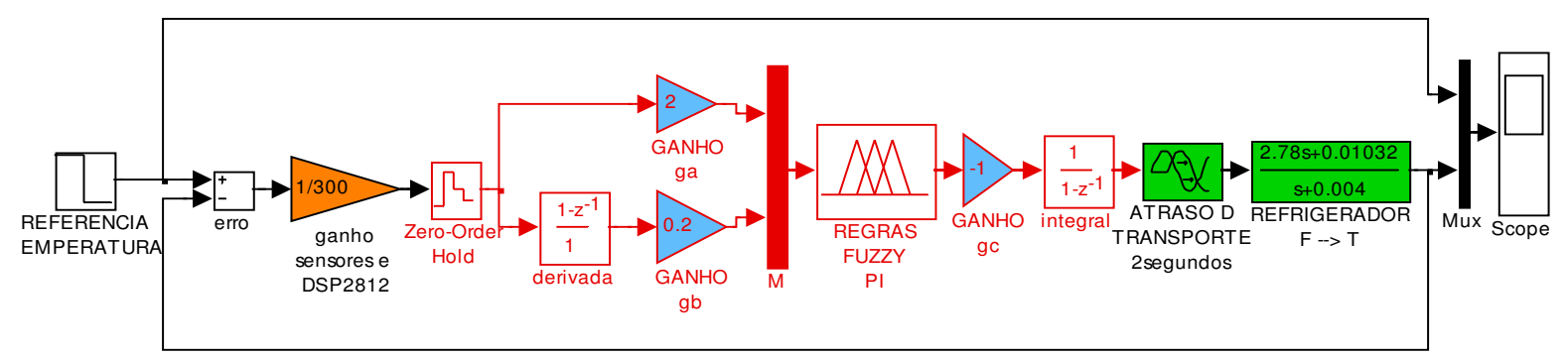

Fig. 7. Simulação do controle de temperatura de refrigerador utilizando compensador PI-Fuzzy. 
bloco denominado ganho, sensores e DSP2812. O ganho $\mathrm{g}_{\mathrm{c}}$ tem sinal negativo justamente para sintetizar a reação inversa do controlador à alteração da variável erro. As variáveis de entrada do bloco Fuzzy são o erro $(e)$ e a taxa de variação do erro (de/dt) conforme discutido na seção $\mathrm{V}$ e a variável de saída é a freqüência $(f)$ de excitação do motor/compressor. $\mathrm{O}$ mesmo número de 5 funções-membro triangulares foi utilizado para todas as variáveis de entrada e saída totalizando 25 regras, conforme ilustra a Figura 8 para a variável erro $(e)$. Os rótulos das cinco funções membro são iguais para todas as variáveis e abreviados por NL (negative large), NS (negative small), ZR (zero), OS (positive small), PL (positive large).

A variável erro $(e)$ abrange a faixa de $-0,075 \mathrm{~V}$ a $0,075 \mathrm{~V}$ a qual define, em graus Celsius, o universo de discurso com as funções membro centradas em $-7,5^{\circ} \mathrm{C},-3,75^{\circ} \mathrm{C}, 0{ }^{\circ} \mathrm{C},+3,75$ ${ }^{\circ} \mathrm{C},+7,5^{\circ} \mathrm{C}$.

A variável taxa de variação do erro $(d e / d t)$ engloba os valores de $-0,025 \mathrm{~V}$ a $0,025 \mathrm{~V}$ os quais definem, em graus Celsius/segundo, o universo de discurso com as funções membro centradas em $-2,5{ }^{\circ} \mathrm{C} / \mathrm{s},-1,25{ }^{\circ} \mathrm{C} / \mathrm{s}, 0{ }^{\circ} \mathrm{C} / \mathrm{s},+1,25{ }^{\circ} \mathrm{C} / \mathrm{s}$, $+2,5^{\circ} \mathrm{C} / \mathrm{s}$. A variável de saída freqüência $(f)$ abrange a faixa de 30 à $70 \mathrm{~Hz}$ sendo as funções membro centralizadas em 30 $\mathrm{Hz}, 40 \mathrm{~Hz}, 50 \mathrm{~Hz}, 60 \mathrm{~Hz}$ e $70 \mathrm{~Hz}$.

A Tabela I apresenta o conjunto de regras que sintetiza a base do conhecimento sobre o controle do sistema. O conhecimento foi estabelecido considerando simetria em relação a diagonal, ou seja, a resposta da planta é considerada igual para excitações positivas e negativas.

Contudo, existe uma pequena diferença na resposta dinâmica para um aumento e um decréscimo de temperatura. Logo, a flexibilidade, intrínseca característica de técnicas Fuzzy fica evidenciada, pois alterando uma regra específica na Tabela I (célula) é possível melhorar, pontualmente, a atuação do controlador. A defuzzificação foi executada usando o método COG (centro de gravidade) o qual é definido por:

$$
f^{c r i s p}=\frac{\sum_{i} b_{i} \int \mu_{(i)}}{\sum_{i} \int \mu_{(i)}}
$$

Sendo $\int \mu_{(i)}$ a área sob a função membro $\mu_{i}, b_{i}$ o centro da função membro e $f^{\text {crisp }}$ o número real (crisp) de saída do bloco Fuzzy.

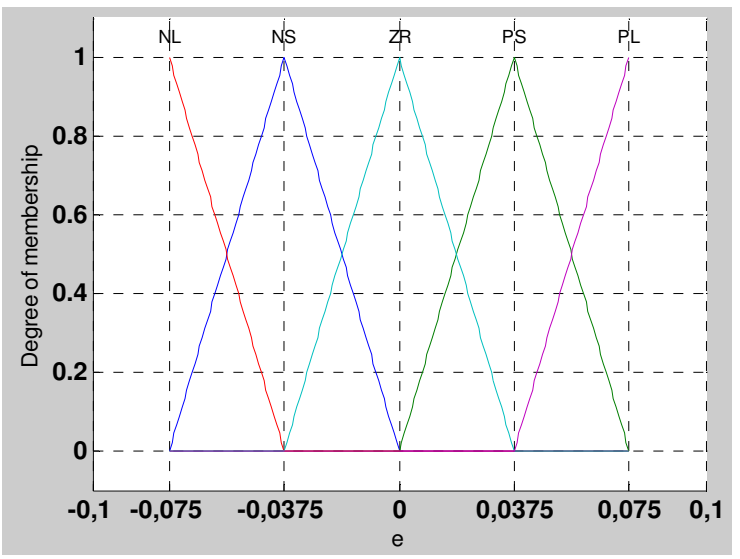

Fig. 8. Funções membro e universo de discurso - variável erro (e).
Contudo, uma vez que as funções membro de saída são triangulares simétricas, no processador TMS320F2812 foi utilizada a seguinte aproximação:

$$
f^{c r i s p}=w\left(h-\frac{h^{2}}{2}\right)
$$

Sendo $w$ a largura da função membro e $h$ a altura do triangulo resultante da inferência Fuzzy do tipo Mandani. Note que a técnica Fuzzy apresenta uma proteção intrínseca para o acionamento do compressor, uma vez que os valores extremos de $30 \mathrm{~Hz}$ e $70 \mathrm{~Hz}$ não são ultrapassados, mesmo que ocorra um erro no controlador.

\section{TABELA I}

Conjunto de regras (base do conhecimento) aplicado na síntese do controlador de temperatura PI Fuzzy.

\begin{tabular}{|c|l|c|c|c|c|c|}
\hline \multirow{2}{*}{\begin{tabular}{c} 
"freqüência \\
compressor" \\
\multicolumn{2}{|c|}{}
\end{tabular}} & \multicolumn{5}{|c|}{ "taxa de variação do erro" de/dt } \\
\cline { 2 - 7 } & NL & NS & ZR & PS & PL \\
\hline \multirow{4}{*}{$\begin{array}{c}\text { "erro" } \\
e\end{array}$} & NL & PL & PL & PL & PS & ZR \\
\cline { 2 - 7 } & NS & PL & PL & PS & ZR & NS \\
\cline { 2 - 7 } & ZR & PL & PS & ZR & NS & NL \\
\cline { 2 - 7 } & PS & PS & ZR & NS & NL & NL \\
\cline { 2 - 7 } & PL & ZR & NS & NL & NL & NL \\
\hline
\end{tabular}

Esta proteção é necessária conforme discutido na subseção IV. Isto ocorre porque o método de defuzzificação COG limita a saída "crisp" nos valores de pico das funções membros da saída.

\section{Efeitos dos ganhos de escalonamento}

A Figura 9 ilustra a resposta para um degrau na referência de temperatura de $2,78 \mathrm{~V}\left(+5{ }^{\circ} \mathrm{C}\right)$ para $2,58 \mathrm{~V}\left(-15{ }^{\circ} \mathrm{C}\right)$ no instante de tempo 120 segundos e os ganhos $g_{a}=g_{b}=g_{c}=1$. O tempo de acomodação obtido foi de aproximadamente 30 segundos e oscilações são observadas na resposta. Logo, para melhorar a resposta dinâmica uma redução de $\mathrm{K}_{\mathrm{P}}$ e aumento de $\mathrm{K}_{\mathrm{I}}$ foram simuladas considerando os novos ganhos $g_{a}=2, g_{b}=0,2$ e $g_{\mathrm{c}}=1$ de acordo com (14) e (15).

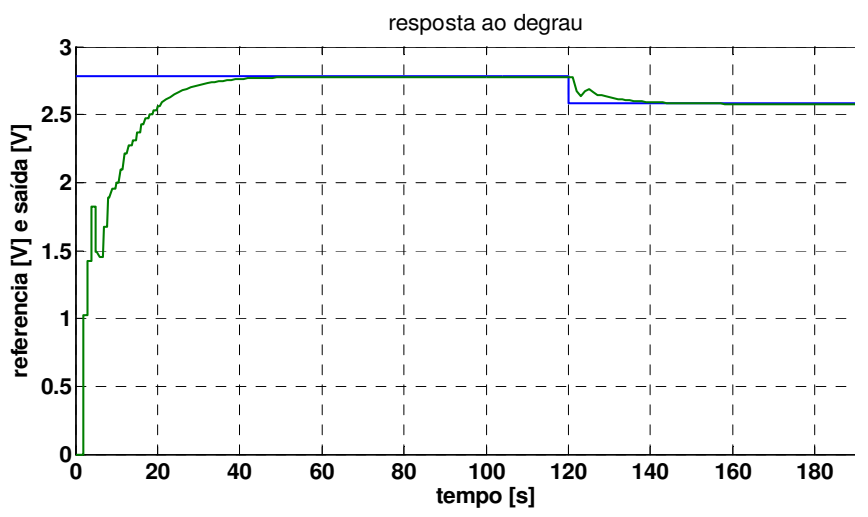

Fig.9. Resposta ao degrau para ganhos de escalonamento iguais a unidade $\left(g_{a}=g_{b}=g_{c}=1\right)$. 


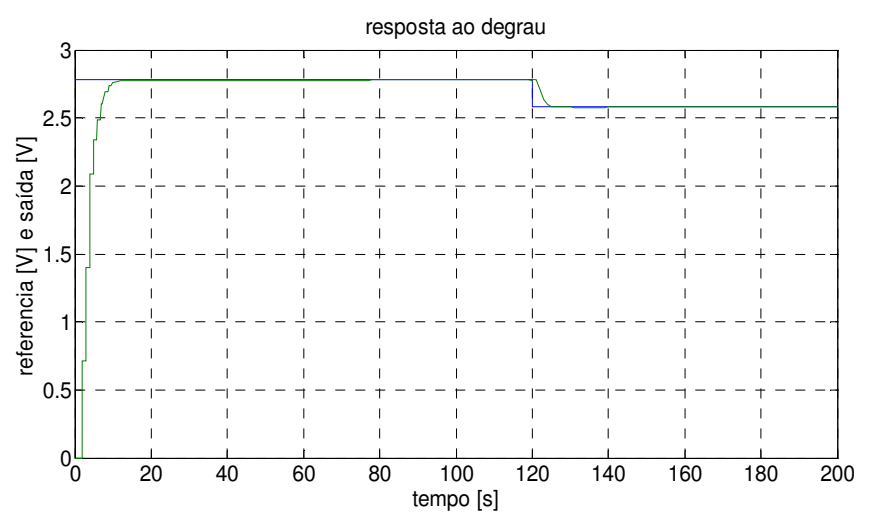

Fig. 10. Resposta dinâmica para ganhos $g a=2, g b=0,2, g c=1$.

A Figura 10 apresenta os resultados para esta nova situação. Fica evidenciado o menor tempo de acomodação (5 segundos) e a melhor resposta dinâmica com os novos ganhos de escalonamento. Estes valores obtidos através de simulação foram aplicados no algoritmo do compensador Fuzzy-PI compilado no processador TMS320F2812.

\section{RESULTADOS EXPERIMENTAIS}

A Figura 11 apresenta a temperatura instantânea no ar dentro do evaporador (ponto de medição - Figura 4). O compensador Fuzzy-PI mantém o valor médio abaixo do valor de $-6^{\circ} \mathrm{C}$ conforme estabelecido na norma ISO7371.

No refrigerador original, controlado por termostato a variação de temperatura nos trocadores de calor atingia uma variação de $18{ }^{\circ} \mathrm{C}$, ao passo que este valor é reduzido para $7^{\circ} \mathrm{C}$ com o compensador Fuzzy-PI. Logo, os trocadores de calor são submetidos a uma menor variação, portanto aumentando o rendimento.

No instante $t_{o}=500 \mathrm{~s}$ a porta do refrigerador foi aberta causando a elevação da temperatura e ativação do controlador Fuzzy. Devido à abertura da porta uma onda de calor invade o gabinete causando uma taxa de variação da temperatura significativa, conforme indica a Figura 11. Ao atingir $+5{ }^{\circ} \mathrm{C}$ o compressor é acionado e em menos de 300 segundos, em $t_{1}$ a temperatura é reduzida para valores próximos a $-12{ }^{\circ} \mathrm{C}$. A rápida resposta decorre do acionamento do compressor a

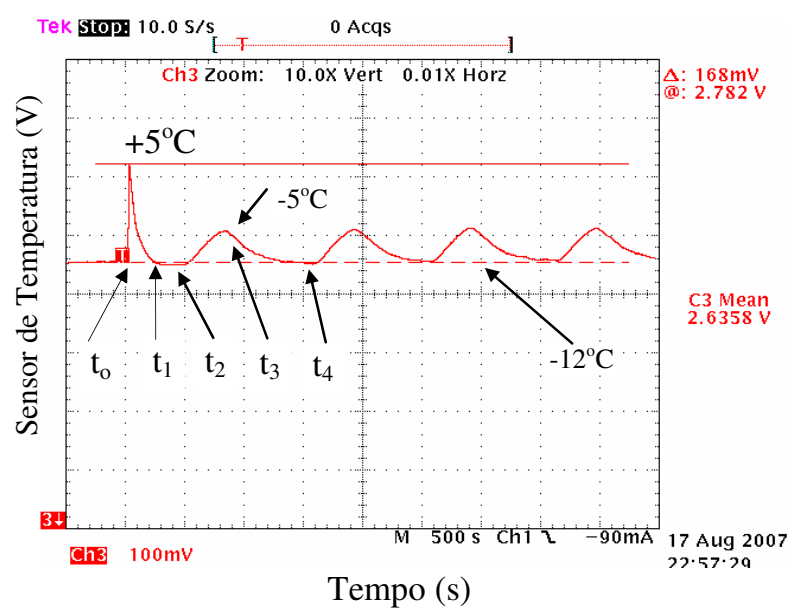

Fig. 11. Temperatura no ar dentro do evaporador (ponto de medição - Figura 4). velocidade máxima de $4200 \mathrm{rpm}(70 \mathrm{~Hz})$. O controlador Fuzzy reduz a velocidade proporcionalmente a diminuição de temperatura. No instante $t_{2}$ o compressor é desligado devido ao baixo valor de temperatura. Logo a seguir a temperatura do gabinete volta a aumentar naturalmente devido à troca térmica com o ambiente. Após 300 segundos (5 minutos), ou seja, em $t_{3}$ a temperatura elevou naturalmente para $-5^{\circ} \mathrm{C}$. Os 5 minutos de parada são programados no DSP para permitir a equalização da pressão no tubo capilar. Transcorridos os 5 minutos o controlador Fuzzy retoma, controlando a velocidade do compressor até que a temperatura retorne ao valor de $-12{ }^{\circ} \mathrm{C}$ no instante $t_{4}$. A partir deste instante o ciclo recomeça, contudo a variação da temperatura é devida somente à troca térmica com o ambiente (porta permanece fechada).

Para a medição do consumo de energia elétrica o equipamento BMI-DRANETZ 7100 (PES) foi utilizado conforme ilustra a Figura 12. Para o período de 1,5 dias de medição (cursores X1 e X2) o consumo contabilizado (considerando os dois enrolamentos) foi de $0,78 \mathrm{kWh} /$ dia. Isto representa uma redução de $9,84 \%$ em relação ao consumo de 0,8567 $\mathrm{kWh} /$ dia registrado para o equipamento original.

Contudo, esta redução de eficiência é um valor indicativo, uma vez que a medição de consumo não foi executada em câmara térmica nas condições exigidas por norma, ou seja, umidade relativa de $50 \%$ e temperatura de $32{ }^{\circ} \mathrm{C}$. Um valor maior que $20 \%$ é previsto se as condições da norma forem atendidas, segundo a literatura.

O conversor de potência foi dimensionado e testado para atender refrigeradores de maior capacidade de refrigeração equipados com compressores de até $1 / 2$ HP. Os resultados experimentais para o conversor com capacitância total de $1880 \mathrm{uF}$, indutância de $3 \mathrm{mH}$ e fornecendo potência de 365 W são indicados na Figura 13.

A Figura 13 ilustra a tensão e corrente na rede para operação em regime, juntamente com a tensão nos capacitores inferior e superior. A tensão total média no link CC é de $414,4 \mathrm{~V}$, mantidos 208,3 V no capacitor superior e 206,1 V no capacitor inferior. A tensão de entrada é de $123,6 \mathrm{~V}$ eficazes para uma corrente de entrada de 3,5 A.

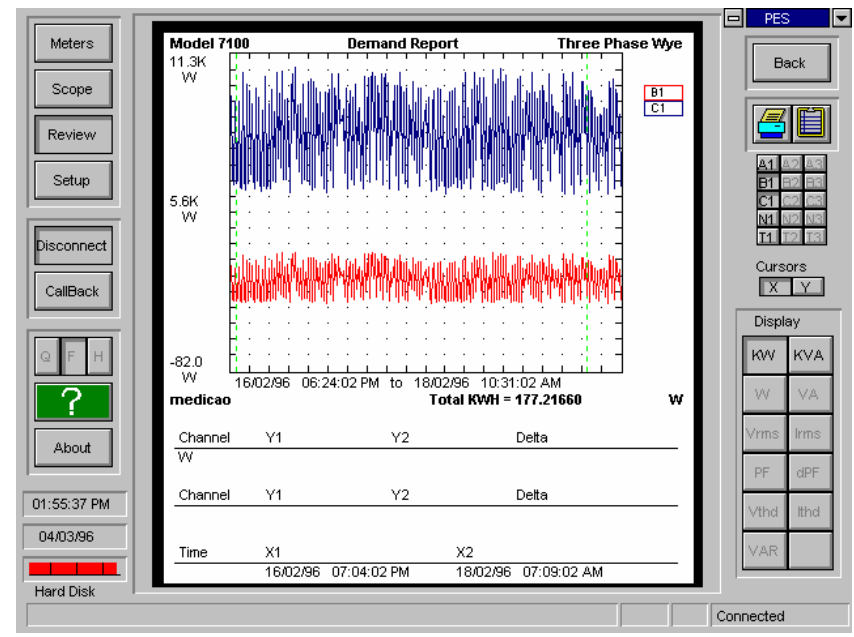

Fig. 12. Consumo de energia - controlador Fuzzy-PI. 


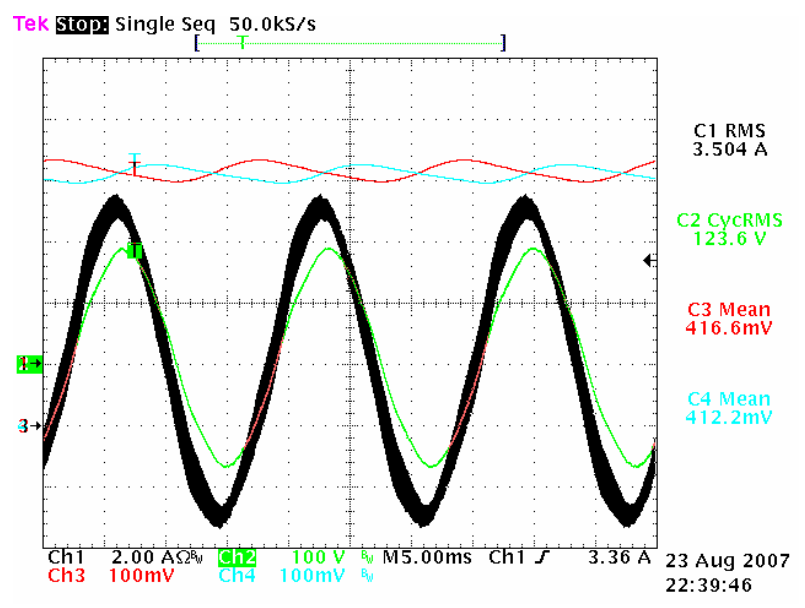

Fig. 13. Tensão (100V/div) e corrente (2A/div) na rede e tensões nos capacitores do link CC (50V/div).

Quando o compressor é desligado pelo controlador Fuzzy o estágio de entrada opera a vazio. O efeito transitório no estágio de entrada e link CC é visualizado na Figura 14. O sistema operava com alto fator de potência quando toda a carga foi retirada abruptamente causando uma oscilação nas tensões dos capacitores do link CC. Os compensadores de tensão e corrente foram implementados no processador com saturação dinâmica do erro proporcional e da ação integral para melhorar a resposta dinâmica em situações com variações abruptas.

O controle escalar V/f foi utilizado no acionamento do motor e, para otimização do conjugado [13], as tensões foram mantidas com defasagem de 90 graus e a razão entre as amplitudes dos enrolamentos igual à relação de espiras, ou seja, $\mathrm{n}=1,29$.

A Figura 15 ilustra as formas de onda de tensão, corrente e potência instantânea observadas no caso original, ou seja, refrigerador conectado à rede elétrica conforme indicado na Figura 2. Neste caso, o fator de potência medido foi de $f p=0,64$, para o qual prepondera um elevado fator de deslocamento, uma vez que os valores percentuais de harmônicos de corrente medidos foram de $3^{\mathrm{a}}(6,2 \%), 5^{\mathrm{a}}(5 \%)$ e $7^{\mathrm{a}}$ $(1,5 \%)$. A distorção harmônica de tensão (THD) estava menor que $0,5 \%$.

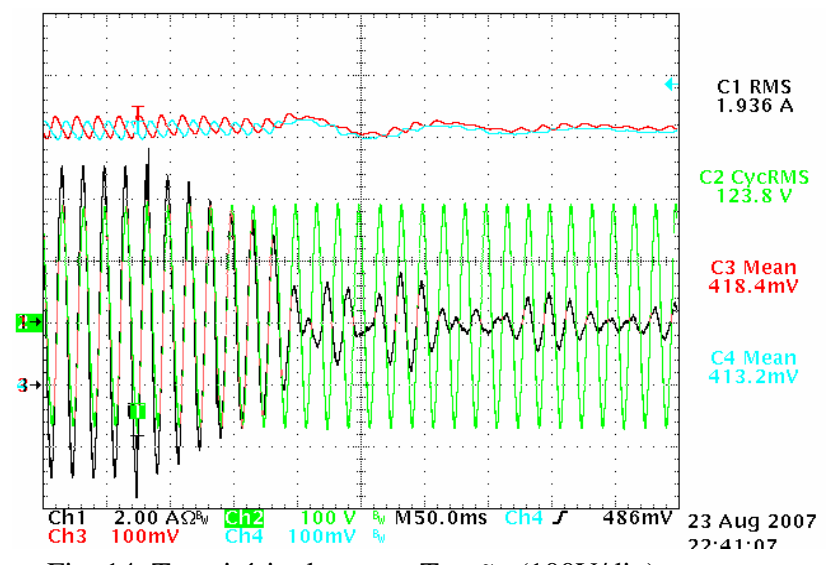

Fig. 14. Transitório de carga. Tensão (100V/div) e corrente (2A/div) na rede. Tensões nos capacitores do link CC (50V/div).

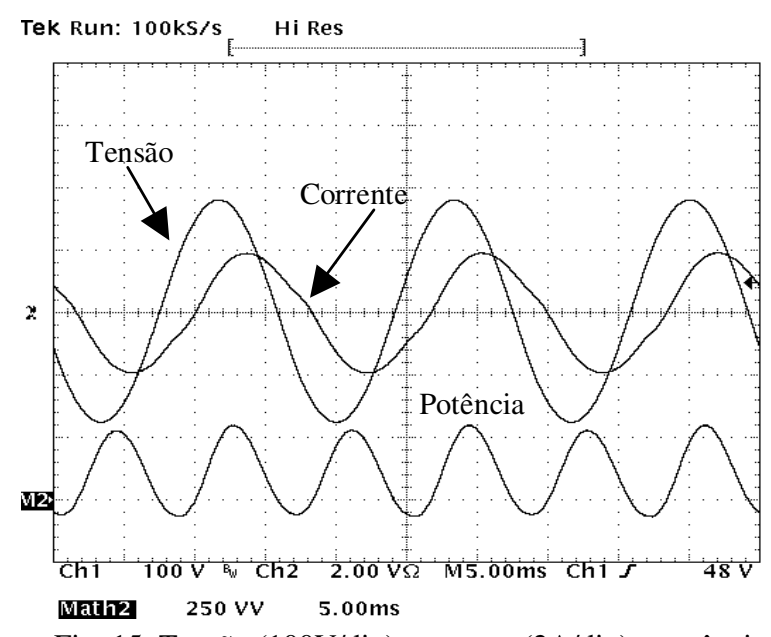

Fig. 15. Tensão (100V/div), corrente (2A/div) e potência (250W/div). Escala horizontal: $5 \mathrm{~ms} / \mathrm{div}$.

Os resultados experimentais, obtidos utilizando o conversor estático de potência ilustrado na Figura 3, permitem obter fator de potência próximo a unidade. Contudo, um filtro é necessário na entrada do conversor para atenuar os harmônicos de alta freqüência devido à comutação dos transistores de potência em $12 \mathrm{kHz}$.

O conversor estático flexibiliza o acionamento, possibilitando que, após a partida do compressor, o enrolamento auxiliar permaneça energizado ou seja totalmente desconectado. Na primeira opção, geralmente a otimização do conjugado eletromagnético é o objetivo em aplicações de motores monofásicos com dois enrolamentos assimétricos [9][13]. A segunda opção é devida em projetos de eficiência energética como no caso do acionamento de compressores, uma vez que o enrolamento auxiliar possui elevada resistência e, portanto ocasiona perdas adicionais quando permanece conectado.

\section{CONCLUSÃO}

Resultados experimentais comprovam o aumento da eficiência energética, em um refrigerador doméstico, obtido através do controle da temperatura do evaporador empregando técnicas Fuzzy.

Do ponto de vista termodinâmico, a opção pela redução da variação da temperatura nos trocadores de calor foi confirmada como sendo uma estratégia apropriada para a redução do consumo de energia elétrica do refrigerador.

Os registros do perfil dinâmico da temperatura indicam que um compensador Fuzzy-PI é adequado para controlar a temperatura do refrigerador a qual tem comportamento peculiar devido à influência de eventos distintos, ou seja, abertura de porta, convecção térmica natural e variação da carga térmica. $\mathrm{O}$ efeito dos ganhos de escalonamento inseridos no compensador Fuzzy-PI foi verificado através de simulação, a qual considerou um modelo de primeira ordem para a planta, estimada a partir do comportamento dinâmico da temperatura.

Os resultados experimentais comprovaram que o conversor eletrônico de potência utilizado é apropriado para garantir simultaneamente alto fator de potência e a mínima tensão no link CC. Esta última é necessária para possibilitar a síntese da tensão eficaz que, uma vez aplicada ao enrolamento 
auxiliar, garante a otimização do conjugado eletromagnético. Em aplicações específicas de eficiência energética, após a partida do compressor, o enrolamento auxiliar deve ser preferencialmente desconectado para reduzir perdas.

\section{AGRADECIMENTOS}

Os autores agradecem a FAPESP, processo número 02/08938-3R, pelo financiamento desta pesquisa e a Whirlpool e International Energy Initiative (IEI/LA) pelo apoio técnico.

\section{REFERÊNCIAS BIBLIOGRÁFICAS}

[1] G. de M. Jannuzzi, G. de C. Queiroz, E. A. Vendrusculo, T. Borges, J. A. Pomilio, "A life-cycle cost analysis (LCCA) for setting energy-efficiency standards in Brazil: The case of residential refrigerators", in Proceedings of the ACEEE Summer Study on Energy Efficiency in Industry, July 2003.

[2] International Organization for Standardization: ISO7371, Household refrigerating appliances - refrigerators with or without low temperature compartment Characteristics and test methods, European Technical Standard.

[3] Instituto Nacional de Metrologia, Normalização e Qualidade Industrial, Tabelas de Consumo/Eficiência Energética. Disponível em www.inmetro.gov.br/consumidor/pbe.asp. Acesso em 2008.

[4] ENERGY-PLUS, Europe's most energy-efficient refrigerators \& freezers. Disponível em: www.energyplus.org/english/products/available. Acesso em 2008.

[5] V. V. Badami, H. Stephanou, "Fuzzy Logic-Based Supervisory Control of Household Appliances", Proceedings of the Sixth IEEE International Conference on Fuzzy Systems, vol. 1, pp. 213-218, July 1997.

[6] M. Mraz, "The design of intelligent control of a kitchen refrigerator", Mathematics and Computers in Simulation - Transactions of IMACS, Elsevier, vol. 56, nº 3, pp. 259-267, June 2001.

[7] G. de M. Jannuzzi, S. Lawaetz e H. Sharon, "Costbenefit analysis of a refrigerator replacement program for low-income households in Brazil", U.S. Agency for International Development (USAID) - Economic Growth, Agriculture and Trade, Order No. PN-ADJ546. Disponível em: http://pdf.usaid.gov/pdf_docs/PNADJ546.pdf._Acesso em 2008.

[8] A. D. Little, "Opportunities for Energy Savings in the Residential and Commercial Sectors with HighEfficiency Electric Motors - Final Report", U.S. Department of Energy - Energy Efficiency and Renewable Energy. Disponível em: http://www.eere.energy.gov/EE/buildings.html. Acesso em 2008

[9] J. Yao, J. Krase e T. A. Lipo, "Design Considerations for Motor-Controller Integration of A Single Phase Induction Motor Packaged Drive", IEEE International
Electric Machines and Drives Conference - IEMDC, vol.2, pp.1239 - 1244, June 2003.

[10]J.T. Boys, A.W. Green, "Current-forced single-phase reversible rectifier"; IEE Proceedings, vol. 136, Pt. B, n ${ }^{\circ}$ 5, September 1989.

[11]F. V. da Silva, Comparação do desempenho de um sistema de refrigeração para resfriamento de líquido, controlado a diferentes modos de controle, Tese de Doutorado, Faculdade de Engenharia de Alimentos FEA/UNICAMP, Brasil, 2003.

[12]H. X. Li, H. B. Gatland, "Conventional Fuzzy Control and Its Enhancement", IEEE Transactions on Systems, Man, and Cybernetics-Part B: Cybernetics, vol. 26, $\mathrm{n}^{\circ}$. 5, pp. 791-796, 1996.

[13]D. G. Holmes, A. Kotsopoulos, "Variable speed control of single and two phase induction motors using a three phase voltage source inverter". Proceedings of the IEEE Industry Applications Annual Meeting Conference, pp. 613-620, 1993.

\section{DADOS BIOGRÁFICOS}

Edson Adriano Vendrusculo, nascido em 06/08/1969, é engenheiro eletricista, mestre e doutor (2001) em Engenharia Elétrica pela Universidade Estadual de Campinas.

Desde 2002 atua como consultor na área de eletrônica industrial e pesquisador colaborador junto a Faculdade de Engenharia Elétrica e de Computação da Unicamp. Realizou estágio de pós-doutoramento junto ao Núcleo Interdisciplinar de Planejamento Energético da Unicamp e foi consultormembro da International Energy Initiative - IEI/LA.

Dr. Vendrusculo é membro da SOBRAEP.

José Antenor Pomilio, nascido em 05/06/1960, é engenheiro eletricista, mestre e doutor em Engenharia Elétrica pela Universidade Estadual de Campinas.

De 1988 a 1991 foi chefe do grupo de eletrônica de potência do Laboratório Nacional de Luz Síncrotron. Realizou estágios de pós-doutoramento junto à Universidade de Pádua e à Terceira Universidade de Roma, ambas na Itália. Foi presidente da Associação Brasileira de Eletrônica de Potência SOBRAEP e membro do comitê administrativo da IEEE Power Electronics Society. Atualmente é editor associado da IEEE Trans. on Power Electronics e da revista Controle \& Automação. É professor Titular da Faculdade de Engenharia Elétrica e de Computação da Unicamp, onde é docente desde 1984.

Dr. Pomilio é membro da SOBRAEP, da SBA, da SBPC, da ABENGE e Senior Member do IEEE. 